\title{
Burn shock and multiple organ failure syndromes
}

\author{
Erkin A. Hakimov, Babur M. Shakirov, Bohodir H. Karabaev", Komil R. Tagaev \\ Burn department of RSCUMA, Samarkand State Medical Institute Samarkand, Uzbekistan \\ E mail address: \\ baburshakirov@yahoo.com(E. A. Hakimov),baburshakirov@yahoo.com(B. M. Shakirov), x.karabaev@yandex.ru(B. H. Karabaev), \\ yorkinkhaydarov@yandex.ru(K. R. Tagaev)
}

\section{To cite this article:}

Erkin A. Hakimov, Babur M. Shakirov, Bohodir H. Karabaev, Komil R. Tagaev. Burn Shock and Multiple Organ Failure Syndromes. Science Journal of Clinical Medicine. Vol. 2, No. 3, 2013, pp. 87-91. doi: 10.11648/j.sjcm.20130203.15

\begin{abstract}
Critical condition, developing in patients with burns during the burn shock more often produce the development of nonspecific reactions in the body, manifested as the system or the organ insufficiency and are determined by the term «syndrome of poly organ insufficiency» (SPOI). The course and treatment of 189 patients with burn shock have been analyzed. With the purpose of investigation all patients with burn shock were subdivided into 2 groups: The first (control) group included 79 patients ( 23 children, 56 adults) and was treated according to traditional antishock infusion-transfusion therapy without inotropic therapy. The second (basic) group included 110 patients ( 33 children, 77 adults) with burn shock and underwent a complex, antishock infusion-transfusion therapy with employment of inotropic and organoprotective therapy depending on dysfunction of vitals and systems. All patients with burn shock underwent general clinical examinations of: cardiovascular and respiratory systems, functions of the lever, kidneys and gastrointestinal tract for revealing of polyorgan insufficiency (POI). The victims of burns - material prove high spread POI in patients with burns, that requires complex, purposeful conservative (local and general) and surgical treatment for its reduction and prevention of unfavorable results.
\end{abstract}

Keywords: Shock, Syndrome of Poly Organ Insufficiency» (SPOI) and Treatments

\section{Introduction}

Thermal trauma remains one of the real problems of modern medicine, because of its heavy clinical course, the difficulties of treating the victims, the high mortality rate, and sometimes unacceptable results of treatment. However, according to the World Health Organization (WHO) data, more than $95 \%$ of fatal fire-related burns in 2002, for example, occurred in low-and middle - income countries [1].

In spite of great success achieved in burn treatment, a lethal outcome among severely burnt patients remains high even in specialized hospitals. Lethality is particularly high in critical (40-50\% of body surface) and extra critical (over $50 \%$ ) deep burns [2-6]. Considerable part of these patients dies during the period of burn shock.

Critical condition, developing in patients with burns during the burn shock more often produce the development of nonspecific reactions in the body, manifested as the system or the organ insufficiency and are determined by the term «syndrome of poly organ insufficiency» (SPOI).

Independent of patient's age consequence of SPOI formation is the following: encephalopathy $\rightarrow$ gastrointestin- al dysfunction $\rightarrow$ acute injury of the lungs $\rightarrow$ cardiovascular dysfunction $\rightarrow \mathrm{DBC} \rightarrow$ Hepatic dysfunction $\rightarrow$ renal dysfunction [7-11].

SPOI dysfunction is very serious: in dysfunction of two organs lethality makes $30-40 \%$, in dysfunction of four or more organs or systems convalescence is practically impossible. However if active treatment of preventive character is provided, the patients life can be saved [12-13].

\section{Patients and Methods}

The course and treatment of 189 patients with burn shock have been analyzed. All of them were treated in Samarkand branch of RSCUMA during the period of 19992009. With the purpose of investigation all patients with burn shock were subdivided into 2 groups.

The first (control) group included 79 patients ( 23 children, 56 adults) and was treated according to traditional antishock infusion-transfusion therapy without inotropic therapy during 1999-2003.

The second (basic) group included 110 patients (33 children, 77 adults) with burn shock and underwent a complex, antishock infusion-transfusion therapy with employ- 
ment of inotropic and organoprotective therapy depending on dysfunction of vitals and systems during 2002-2009.

All patients with burn shock underwent general clinical examinations of: cardiovascular and respiratory systems, functions of the lever, kidneys and gastrointestinal tract for revealing of polyorgan insufficiency (POI).
Clinical analyses of blood and urine were carried out. Taking into account the traumas of this type, careful control of arterial pressure level in dynamics, temperature reaction, respiratory and pulse rate, measuring of hourly and daily dieresis were of particular significance.

Table 1. Structure of the organ dysfunction in patients with burn shock (control group)

\begin{tabular}{|c|c|c|c|c|}
\hline \multirow{2}{*}{ Systems } & \multicolumn{3}{|c|}{ Degrees of shock } & \multirow{2}{*}{$\begin{array}{l}\text { Total } \\
\mathrm{n}=79\end{array}$} \\
\hline & $\mathrm{In}=42$ & II $n=28$ & III $n=9$ & \\
\hline Respiratory & $20(47,6 \%)$ & $27(96,4 \%)$ & $8(88,9 \%)$ & $55(69,6 \%)$ \\
\hline Cardiovascular & $23(54,8 \%$ & $25(89,3 \%)$ & $8(88,9 \%)$ & $56(70,9 \%)$ \\
\hline $\mathrm{CNS}$ & $19(45,2 \%)$ & $25(89,3 \%)$ & $7(77,7 \%)$ & $51(64,6 \%)$ \\
\hline Kidneys & $21(50,0 \%)$ & $26(92,5 \%)$ & $7(77,7 \%)$ & $54(68,4 \%)$ \\
\hline Liver & $20(47,6 \%)$ & $17(60,7 \%)$ & $5(55,5 \%)$ & $42(53,2 \%)$ \\
\hline GIT & $18(42,8 \%)$ & $26(92,5 \%)$ & $6(66,6 \%)$ & $50(63,3 \%)$ \\
\hline Hemostasis & $5(11,9 \%)$ & $10(35,7 \%)$ & $6(66,6 \%)$ & $21(26,6 \%)$ \\
\hline
\end{tabular}

Table 2. Structure of the organ dysfunction in patients with burn shock (basic group)

\begin{tabular}{|c|c|c|c|c|}
\hline \multirow{2}{*}{ Системы } & \multicolumn{3}{|c|}{ Степени шока } & \multirow{2}{*}{$\begin{array}{l}\text { Всего } \\
\mathrm{n}=110\end{array}$} \\
\hline & $\mathrm{In}=60$ & II $n=39$ & III $n=11$ & \\
\hline Respiratory & $2(35,0 \%)$ & $20(51,3 \%)$ & $10(90,9 \%)$ & $51(46,4 \%)$ \\
\hline Cardiovascular & $30(50,0 \%)$ & $22(56,4 \%)$ & $9(81,8 \%)$ & $61(55,5 \%)$ \\
\hline $\mathrm{CNS}$ & $16(26,7 \%)$ & $21(53,8 \%)$ & $8(72,7 \%)$ & $45(40,9 \%)$ \\
\hline Kidneys & $16(26,7 \%)$ & $19(48,7 \%)$ & $8(72,7 \%)$ & $43(39,1 \%)$ \\
\hline Liver & $10(16,7 \%)$ & $15(38,5 \%)$ & $7(63,6 \%)$ & $32(29,1 \%)$ \\
\hline GIT & $9(15,0 \%)$ & $17(43,6 \%)$ & $6(54,5 \%)$ & $32(29,1 \%)$ \\
\hline Hemostasis & $7(11,7 \%)$ & $6(15,4 \%)$ & $6(54,5 \%)$ & $19(17,3 \%)$ \\
\hline
\end{tabular}

\section{Results}

The most severe disturbances in the shock period are observed in cardio respiratory system of patients with burns. Impairments of central hemodynamic due to extra and intracardial distress are characteristic for acute period of burn trauma first of all. There is traditional orientation in combustiological practice on routine criteria in shock diagnosis - RSS, AP, Allgover index and clinical estimation of the victim's condition.

The study of body systems impairments in the observed patients with burn shock showed (Table 1, 2) that POI occurred in all patients with burns $-189(100 \%)$, with this in the majority of cases $-150(79,4 \%)$ combination of three and more organs and systems dysfunctions was revealed.

In $96(50,8 \%)$ patients with POI syndrome and burn shock, damage of central nervous system as encephalopathy syndrome, (ES) was revealed, it proves its principle role in regulation of vital function of the body. High capability of the central nervous system, for injuries develops in patients with burns the conditions for dysfunctions and then decompositions of the systems

providing vital activity in the body and further development of POI syndrome in patients with burn shock.

Insufficiency $(47,8 \%)$ in the central nervous system was manifested in the majority of cases as intoxication or alcohol - intoxicated delirium, impairment of consciousness (excitation, inhibition, sopor, coma).

With the same high frequency impairment of respiratory system - $106(56,1 \%)$ and gastrointestinal tract $-82(43,4 \%)$ developed in patients with burns, resulting in hypoxia, impairment of metabolic processes and water - electrolyte metabolism which are most significant in development of severe course in burn shock.

High incidence of abnormalities in the central nervous system, respiratory system and GIT in the structure of polyorgan insufficiency syndrome proves a response of the patient's body of the same type to the effect of the burn trauma.

Syndrome of impairment of cardiovascular system usually developing is early stages of burn shock were revealed in more than half - $96(50,8 \%)$ patients and it had hypoxic - metabolic genesis.

Syndrome of renal inefficiency developed in 97 (51, 3\%) patients and hepatic insufficiency in $74(39,2 \%)$ patients.

The most actual in POI peak is syndrome of intestinal insufficiency (SII).

Insufficiency of GIT organs was marked by toxic paresis of the stomach and intestine, development of acute erosions and ulcers of the esophagus, stomach, duodenum, small intestine often complicated by bleeding (12 patients).

Preventive oesophagogastroduodenoscopies were performed in 17 patients in 1-3 days and in the cases of gastroduodenal bleedings additionally, depending on indica- 
tions.

Intestinal tube is a target organ in the condition of burn shock. Its reaction to ischemia is the earliest and it is, as well as burn injury, entry for infection. The feature of the thermic trauma is complete or partial anorexia that resulted in cachexy in a short period in early stages of combustiology development and developing hyper metabolism caused unhealing, for a long time, injuries and stable course of polyorgan dysfunction.

Analyses of intestinal dysfunction manifestations in patients with severe thermic trauma made it possible to separate 4 main symptoms: repeated vomiting (47\%), development of Kurlings stress ulcers (19\%), gastrointestinal tracts paresis with appearance of congestive discharge from the stomach $(25 \%)$ and repeated watery stool $(9 \%)$. These symptoms most often developed in children, aged 8-15 years $(63,2-66,6 \%$ of these patients had upper mentioned disturbances).

As a rule the incidence of intestinal damage depends on severity of injuries. Thus, damage of the body surface to $20 \%$ is accompanied by intestinal dystonia in $37,5 \%$ of cases, and burns covering more than $40 \%$ of the body surface result in gastrointestinal tract dysfunction already in $58,6 \%$ of cases.

Duration of shock for more than 20 hours and correspondingly more severe reperfusion damage of tissues result in direct impairment of gastrointestinal tract. In duration of shock for less than 20 hours the symptoms of gastrointestinal tract dysfunction were not revealed but in case with duration of shock for more than 60 hours pathological symptoms were revealed in $49,3 \%$ of patientients.

The regularity is noted: with increase of shock duration severity of gastrointestinal impairment is more marked. It is accompanied by repeated vomiting; Kurling's ulcers with bleedings are developed.

Therefore, severity of burn trauma determines the incidence of digestive and absorbing impairments. In its turn, presence of intestinal dysfunction may be the evidence of severe course of burn disease.

According to our findings the feeding probe normalized volemic parameters and proteins, circulating in blood, and also contributes to early normalization of GIT functions. Enteral infusion made it possible to decrease the volume of infusion - transfusion therapy on $30 \%$ and to decrease the blood expense on $17 \%$.

The role of the intestine is important for the origin of polyorgan insufficiency; the mechanism of its development is shown on the scheme. Together with this it should be noted that realization of internal feeding in the postagressive period has certain difficulties because GIT is one of the main targets of postagressive reaction of any origin (wound, burn, operation, etc.) due to the effect of numerous both primary and secondary factors, such as:

- centralization of blood circulation, resulting in circulatory hypoxia on GIT level, hypoergoza and impairments of not only high intensive regenerative process on the level of mucous membrane but all factors of its defence;
- frequent development of intestinal paresis, taking place in these cases, accompanied by water electrolytic impairments

- ascendant dysbiosis with phenomena of endogenic microbe intoxication and transitional translocation of microbes into the blood;

- dysfunction of regulatory hormones and peptide products, developed in the duodenum, that destroys entro - enteral and entro - visceral regulatory interconnections;

- impairment of intracavitory and membraneous digestion as well as absorption.

The upper indicated total combination of facts may result in development of acute intestinal inefficiency (AII), which is manifested by combined impairments of motor, secretory, digestive and absorptive functions, first of all of the small intestine and its exclusion from metabolic processes. In AII development three stages are distinguished:

The I stage (intestinal stasis) - acute motor impairments without accumulation of gases and fluid in the intestine, with this, microcirculation, absorption and epitheliocytes structure are preserved;

The II stage (intestinal paresis) - motor impairment is accompanied by accumulation of gases and fluid in the intestine and there is also malabsorption, venous stasis and changes in epitheliocytes;

The III stage (dynamic intestinal obstruction and enterorrhagia), marked impairments of arterial and venous microcirculation, progressing hypoxia and deep dystrophy of enterocytes, extension of intestinal loops, absence of peristalsis, increasing deficiency of water, clectrolytes and labile proteins.

In connection with this it should be noted that the main preventive measures in AII are:

- optimal and constant decompression of the stomach and intestine;

- intestinal lavage and active enterosorption;

- use of regulatory (amtizol, cytochrom - C) and substrate (succinat, fumarat, glutamine, succinic acid) antioxidants;

- early administration of enteral feeding.

Hepatic dysfunction or post-shock hepatitis is one of manifestations of POI in patients with burns. Its incidence also varies widely depending on age, however predominant age in this case is the period of adolescence that is due to social cases (the majority of suffered adolescent patients take alcohol, toxic and narcotic substances).

The main form of pulmonary clinical pathology in burn disease is pneumonia which is not only most frequent but the most severe complication of burn disease. In a number of cases it is involvement of the lungs into the process that is leading in pathogenesis. The incidence of pneumonia according to the date of L.M. Klyachkin et al. makes $20 \%$ of died patients in the shock period. Of them $72 \%$ died in the period between $4-45$ days and $83 \%$ in later terms. With this, early diagnosis of pneumonia in burn disease is very important. 
Taking into consideration a high risk of pneumonia development in burn disease, carrying out preventive measures in the period of shock is of great significance and includes antishock measures, administration of calcium antagonists (finoptin, corinfar), curantil, heparin, i.e. preparations improving microcirculation in addition to antibioticotherapy.

The most severe manifestation of POI is development of acute respiratory distressive syndrome (ARDS). ARDS is nonspecific injury of the lungs, associated with impairment of the endothelium by active mediators, resulting in progressing oxygen depending hypoxia due to decrease of pulmonary compliance, blocking of oxygen diffusion through alveolar - capillary membrane, venous - arterial blood shunting and requiring AVL and other methods of oxygen- transport blood function correction.

The cardiovascular system mostly affected in burn shock patients is the main link in pathogenesis in oxygen transport disturbance. Being in close connection with respiratory system, cardiovascular system is responsible not only for its pump function. The basic disturbance of the cardiovascular system results both in delivery and consumption of oxygen.

Hemodynamic profile of each separate patient is determined by interconnection of a number of factors: background condition and the type of blood circulation system (hypokinetic ukinetic, hyperkinetic), by severity of trauma and duration of pre - hospital period, by the volume and character of previous therapy, depth and localization of the burn.

Renal dysfunction of patients with burn trauma is on the third place $-47,3 \%$ of suffered patients. Suppression of excretory function of kidneys makes it difficult to keep the normal level of hydrogen ions concentration in the blood.

Delay of fluid in the body (in the intestinal tissue) results in decrease of hydrogen ions excretion with urine, disturances of KOC are developed. In a mild corse of a shock shifts in KOC system are limited by increase of basis deficiency by some decrease of carbonic acid content in blood in normal $\mathrm{pH}$ level, but in more severe courses buffer system are not able to compensate the increase of hydrogen ions concentration and $\mathrm{pH}$ shifts to the acidic side occurs. The more severe is a shock the more marked is the decrease of alkaline reserve and more marked acidosis. The degree of KOC change is not only the index of burn shock. All metabolic processes in the body being fermentative depend on $\mathrm{pH}$ level in the blood.

At present pathogenetic role of intensification of free radical peroxide oxidation of lipids in many pathological processes is proved, particularly in patients being in "critical" situation. Their free - radical processes have on intensive course, and protective mechanisms directed on joining of toxic metabololites are decreased.

The body toxins are able to be inductors of there processes. Their role in increase of general peripheral resistance of vessels and disturbance of microcirculation and also in impairment of contractive ability of the myocar- dium, in decrease of cardiac discharge is known. Accumulation of peroxides is favorable for thombogenesis, it contributes to disseminated intravascular clotting of blood, taking place in the process of shock.

In spite of success, achieved in treatment of burn shock, the problem of prevention and treatment of its numerous complications, particularly burn oliguria is actual.

Hourly diuresis is the basic control of efficacy of antishok measures and rehydration. In order to measure it, catheter is inserted into the bladder and is left there until the patient will be taken out of shock. Amount of urine discharge is hourly noted.

Kidney is one of the target organs, affected in first hours after burn injury. Prevention of shock kidney development must be one of the main problems of burn shock intensive therapy.

Disturbance of blood supply in kidneys and blood circulation in glomes due to hypovolemia, microthrombogenesis in glomerular vessels and deposition of fibrin are very significant in the development of this complication.

In addition to hemotehology and hemocoagulation increased vascular permeability and edema associated with it may be of definite importance in renal blood circulation disturbance.

With the aim to decrease vascular permeability, edema and consequently to improve renal blood supply we used glucose - novocaine mixture.

Acute renal insufficiency (45\%) was diagnosed on the basis of disturbances of renal excretory functions, manifested as oliguria or anuria and rarely as polyuria with impairment of renal concentrational function, increase of urea and creatine indexes in blood serum.

Burn shock is a pathological process, associated with plasmorhagia, decrease of heart ability, impairment of vascular regulation, microcirculation, and metabolic impairment. Severity of patients condition, marked POI, the course of the inflammatory process in the wound depend on the condition of microcirculation both in the areas of burns trauma and in vitals.

Increase of blood coagulation properties, slowing down of blood flow, pathological changes in vascular wall, increase of blood viscosity, aggregation of blood corpuscles, marked disturbances of central and peripheral hemodynamics and other factors may result in severe injuries in the blood coagulation system in burn shock.

In its turn disturbances in the system of regulation of aggregate condition of blood (RACB) may effect microcirculatory perfusion.

In estimation of hemostasis system condition in $95 \%$ patients generally accepted indexes were taken into account: amount of thrombocytes, activated partial thrompoplastin time (APTT), general fibrinogen, prothrombin time (according to KVIR), prothrombin index. The patients received improved intensive therapy, including respiratory, inotropic, oxygen support according to indexes. 


\section{Conclusions}

The above - mentioned material prove high spread POI in patients with burns, that requires complex, purposeful conservative (local and general) and surgical treatment for its reduction and prevention of unfavorable results.

\section{References}

[1] WHO Report. The Injury Chart Book: a graphical overview of the global burden of injuries. 2002. p. 27-34.

[2] Herndon D.N. Total burn care // 2nd edition, W.B. Saunders; 2001

[3] Matveenko A.V. et. al. Burn shock. // Collection of scientific works of the I Congress of Russian Combustiologists «World without burns», Moscow, October 17-21, 2005. - P. $69-70$.

[4] Daltroy L., Liang M., Phillips C. et al. American Burn Asssociattion / Shriners Hospitals or Children burn outcomes questionnaire: construction and psychometric properties. J. Burn Care Rehabil 2000; 21:29-39.

[5] Sheridan R., Hinson M., Liang M. et al. Long-term outcome of children surviving massive burns. JAMA 2000; 283:6973.

[6] Yen K.L., Bank D.E., O Neill A.M. et al. Household oven doors: a burn hazard in children. // Arch. Pediatr. Adolesc. Med. 2001; 155:84-6.
[7] Alexeev A.A. et. al. Systemic inflammatory reaction in patients with severe burns. // Collection of scientific works of the III Congress of Russian Combustiologists «World without burns», November 15-18, 2010. - P. 53-54.

[8] Krilov K.M. et.al. The role of wound infection in the development of system inflammatory reaction in patients suffered from severe thermic trauma // First medical aid. Proceedings, International Conference on "The Actual Problems of Thermic Trauma", June 20-22, Saint - Petersburg, 2006. P. - 61-62.

[9] Sakka SG, Bredle DL, Reinhart K, Meier-Hellmann A: Comparison between intrathoracic blood volume and cardiac filling pressures in the early phase of hemodynamic instability of patients with sepsis or septic shock. J Crit Care 1999, 14:78-83.

[10] Kramer GC, Lund T, Beckum O: Pathophysiology of burn shock and burn edema. In Total Burn Care. 3rd edition. Edited by Herndon DN. Philadelphia: Saunders Elsevier; 2007:93-104.

[11] Luce P.V. Acute lung injury and the acute respiratory distress syndrome // Crit. Care Med. 1998, vol. 26, № 2, p. 369 -376 .

[12] Baue A. "Multiple progressive or sequentiel systems failure" // Arch. Surg., 1975.-N.11.-P.779.

[13] Carrico C.J., MeakinsJ.I., Marshall J.C. et al. "Multiple organ failure syndrom" // Arch. Surg., 1986.-Vol.121.-P.196208. 